\title{
CONSERVAÇÃO PÓS-COLHEITA DE MANGABA (Hancornia speciosa Gomes)
}

\author{
Postharvest conservation of mangaba (Hancornia speciosa Gomes)
}

\author{
Marcelo Augusto Gutierrez Carnelossi ${ }^{1}$, Waleska Fernanda Ferreira Toledo ${ }^{2}$, \\ Daniella Cecília Lemos Souza ${ }^{2}$, Moema de Lima Lira ${ }^{3}$, Gabriel Francisco da Silva \\ Vahideh Rabani R. Jalali ${ }^{3}$, Pedro Roberto de Almeida Viégas ${ }^{4}$
}

\begin{abstract}
RESUMO
Conduziu-se o presente trabalho com o objetivo de caracterizar física e quimicamente os frutos da mangaba quando estão "de caída" ou "de vez" e estudar o efeito de diferentes temperaturas de armazenamento sobre a conservação póscolheita desse fruto. Os frutos foram selecionados e armazenados em câmaras frias nas temperaturas de 6,18 e $25 \pm 2^{\circ} \mathrm{C}$. Durante o período de armazenamento, foram retiradas amostras a cada 1, 3, 5, 7 e 9 dias, para determinações dos teores de vitamina $\mathrm{C}, \mathrm{pH}$, acidez titulável e sólidos solúveis ( ${ }^{\circ} \mathrm{Brix}$ ), atividade de água, condutividade, resistividade e difusividade. Verificou-se que mangabas "de caída" apresentaram teores de vitamina $\mathrm{C}$ e sólidos solúveis superiores aos frutos "de vez". $\mathrm{O}$ armazenamento a $6^{\circ} \mathrm{C}$ foi eficiente na manutenção das características físico-químicas de mangabas "de vez", ao passo que, para mangabas "de caída", as características físico-químicas foram mantidas por um período de até três dias, quando submetidas às temperaturas de $18^{\circ} \mathrm{C}$ e $25^{\circ} \mathrm{C}$. Observaram-se diminuição da atividade de água e aumento da condutividade térmica da mangaba durante o período de armazenamento refrigerado. Assim, a condutividade térmica, a resistividade e a atividade de água podem ser utilizadas como parâmetros de acompanhamento pós-colheita dos frutos de mangaba, quando armazenados sob refrigeração.
\end{abstract}

Termos para indexação: Armazenamento, frutos tropicais, Hancornia speciosa.

\begin{abstract}
The present work had the objective of characterizing physico-chemical parameters of the mangaba fruits, either "fallen" or "of the time", and to study the effect of the storage of mangaba (Hancornia speciosa Gomes) at different temperatures on its postharvest conservation. The fruits were selected and stored in cold chambers at 6,18 and $25 \pm 2^{\circ} \mathrm{C}$. During storage, samples were removed at 3,5, 7 and 9 days, for determination of vitamin $\mathrm{C}$ content, $\mathrm{pH}$, total acidity and soluble solids ( $\left.{ }^{\circ} \mathrm{Brix}\right)$ content, water activity, conductivity, resistivity and difusivity. It was verified that "fallen" mangabas presented vitamin $\mathrm{C}$ and solid soluble contents superior to the "of the time" fruits. The storage at $6^{\circ} \mathrm{C}$ was efficient in keeping the physico-chemical properties of "of the time" mangabas, whereas for "fallen" mangabas the physico-chemical properties were maintained for a period of up to three days when submitted to temperatures of 25 and $18^{\circ} \mathrm{C}$. A fall of the water activity and increase of the thermal condutivity of mangaba during the period of cold storage was observed. Thus, the thermal conductivity, the resistivity and the water activity can be used as parameters to follow postharvest fruits of mangaba when stored under refrigeration.
\end{abstract}

Index terms: Storage, tropical fruits, Hancornia speciosa.

(Recebido para publicação em 3 de outubro de 2003 e aprovado em 1ํ de junho de 2004)

\section{INTRODUÇÃO}

O Nordeste brasileiro apresenta condições climáticas favoráveis ao cultivo de muitas espécies frutíferas de clima tropical, o que é evidenciado pela expressiva diversidade de espécies nativas encontradas na região, ao lado de outras, exóticas, introduzidas de ecossistemas equivalentes e que se adaptaram bem, comportando-se de modo semelhante ao do material nativo, a exemplo da jaqueira, mangueira, entre outras.
A mangabeira (Harconia speciosa Gomes), frutífera da família das apocináceas, é uma árvore originária do Brasil. Ocorre nas regiões de vegetação aberta, como cerrados, tabuleiros arenosos, chapadas e caatingas. A colheita dos frutos é iniciada normalmente em novembro ou dezembro e se estende até os meses de maio ou junho (VIEIRA NETO, 2002). A qualidade dos frutos colhidos depende das condições de desenvolvimento, as quais influenciam a vida útil pós-colheita. Colheitas realizadas antes de os frutos atingirem

\footnotetext{
1. Professor-doutor do Departamento de Engenharia Química/UFS, Av. Marechal Rondon s/n, Jd Rosa Elze 49100-000 - São Cristóvão, SE, carnelossi@ ufs.b

2. Iniciação Científica Estudantes de Graduação, Engenharia de Alimentos/UFS

3. Professores-doutores do Departamento de Engenharia Química/UFS.

4. Professor-doutor do Departamento de Agronomia/UFS.
} 
completa maturação fisiológica prejudicam o seu processo de amadurecimento, afetando a sua qualidade. Por outro lado, a colheita de frutos totalmente maduros reduz sua vida útil, dificulta o seu manuseio e transporte, devido a sua baixa resistência física, causando perdas quantitativas e qualitativas (CHITARRA e CHITARRA, 1990).

Uma das técnicas mais eficientes para aumentar a durabilidade de frutos e minimizar as perdas póscolheita é o armazenamento à baixa temperatura. $\mathrm{O}$ uso da refrigeração é utilizado para diminuir a taxa respiratória, a perda de água e retardar o amadurecimento dos frutos (PAULL, 1994). O armazenamento adequado é um dos pontos críticos para o sucesso da comercialização de frutos tropicais.

Para estudo da qualidade dos frutos, podem ser adotados vários parâmetros, sejam eles físicos ou químicos. Esses parâmetros geralmente são influenciados pelos seguintes fatores: edafoclimáticos, cultivar, época e local de colheita, tratos culturais e manuseio na colheita e pós-colheita, e variam em função do destino do fruto e das exigências do mercado consumidor (FAGUNDES e YAMANISHI, 2001).

Os estudos das propriedades físicas de frutos têm sido um fator importante para determinar as condições de conservação em unidades de armazenamento refrigerado (SWEAT, 1974; RIBEIRO et al., 2002) Entretanto, uma das grandes limitações ao uso dessas propriedades reside na grande falta de informação sobre as propriedades térmicas de produtos alimentícios (RIBEIRO et al., 2002).

Com o presente trabalho objetivou-se caracterizar física e quimicamente os frutos da mangaba do tipo "de caída" ou "de vez" e estudar o efeito do armazenamento da mangaba, em diferentes temperaturas, sobre a sua conservação pós-colheita.

\section{MATERIAL E MÉTODOS}

O estudo foi desenvolvido no Laboratório de Tecnologias Alternativas (LTA) do Departamento de Engenharia Química da Universidade Federal de Sergipe. Os frutos utilizados foram adquiridos no município de Itaporanga D'Ajuda/SE e transportados em caixas plásticas para o LTA, sendo todo transporte realizado à temperatura ambiente. No laboratório, foram selecionados dois tipos de frutos, os de "de caída", que são frutos caídos da planta-mãe, coletados no chão, com coloração amarela e com grau máximo de desenvolvimento; e os "de vez", frutos totalmente desenvolvidos, apresentando coloração esverdeada para amarelada, colhidos direta- mente da planta-mãe (VIEIRA NETO, 2002). Foi dada preferência àqueles que apresentaram melhor aparência externa, ou seja, casca íntegra, sem injúrias mecânicas, manchas e rachaduras.

Feita a seleção dos frutos, esses foram acondicionados em bandejas de poliestireno com capacidade para $500 \mathrm{~g}$ de frutos. As amostras foram armazenadas em câmaras frias, à temperatura de 6,18 e $25 \pm 2^{\circ} \mathrm{C}$. Frutos armazenados a $25^{\circ} \mathrm{C}$ foram utilizados como controle. Durante o período de armazenamento, foram retiradas amostras a cada $1,3,5,7$ e 9 dias, para determinação dos teores de vitamina $\mathrm{C}, \mathrm{pH}$, acidez total titulável e sólidos solúveis ( ${ }^{\circ}$ Brix), atividade de água, condutividade térmica, resistividade térmica e difusividade.

Os teores de vitamina $\mathrm{C}$ foram determinados de acordo com a metodologia proposta pela American Official Analysis of Chemistry (AOAC), com adaptações (CARNELOSSI, 2000). A vitamina $\mathrm{C}$ foi expressa em $\mathrm{mg}$ Vitamina $\mathrm{C} 100 \mathrm{~g}^{-1}$ matéria fresca (MF).

$\mathrm{O}$ pH foi determinado no filtrado obtido a partir da homogeneização de $10 \mathrm{~g}$ de polpa dos frutos em 10 $\mathrm{mL}$ de água com areia lavada, para aumentar a superfície de contato do tecido com a solução, otimizando o processo de trituração e extração, filtrado em duas camadas de gase. O filtrado foi transferido com água para béquer, sendo o volume completado para $100 \mathrm{ml}$ com água destilada e o pH determinado em potenciômetro.

A acidez total titulável foi obtida por titulação do filtrado, sob agitação, com $\mathrm{NaOH} 0,1 \mathrm{~N}$, usando-se fenolftaleina a $1 \%$ como indicador. Os resultados foram expressos em porcentagem de ácido cítrico.

O conteúdo de sólidos solúveis totais $\left({ }^{\circ} \mathrm{Brix}\right)$ foi determinado de aproximadamente $2 \mathrm{~mL}$ de extrato, obtido com uso de uma prensa manual, a partir de aproximadamente $5 \mathrm{~g}$ da polpa dos frutos. Os teores de sólidos solúveis foram determinados com o auxílio de um refratômetro Abbé tipo WYA (2WA-J, BIOBRIX).

A atividade de água foi determinada utilizando o aparelho Aqualab (DECAGON). Foram coletados $2 \mathrm{~g}$ de frutos e colocados diretamente nas células de análise do aparelho.

As propriedades térmicas, condutividade, resistividade e difusividade foram determinadas utilizando-se um sensor térmico, o KD2 (DECAGON), sendo a condutividade térmica expressa em $\mathrm{W} \mathrm{m}^{-1}{ }^{\circ} \mathrm{C}^{-1}$; a difusividade, em $\mathrm{mm}^{2} \mathrm{~s}^{-1}$, e a resistividade térmica, em ${ }^{\circ} \mathrm{C} \mathrm{W}^{-}$ 1 .

O delineamento experimental utilizado foi o inteiramente casualizado, montado em esquema fatorial 2 x 3 x 5 (dois tipos de frutos, "de vez" e "de caída"; três temperaturas de armazenamento 6,18 e $25^{\circ} \mathrm{C}$ e cinco 
épocas de avaliação 1, 3, 5, 7 e 9 dias), com três repetições cada tratamento. A unidade experimental constou de uma bandeja contendo $500 \mathrm{~g}$ de frutos. Os dados foram expressos como médias. Teste Tukey, a 5\% de probabilidade, foi utilizado para detecção de diferenças de médias entre os tratamentos.

\section{RESULTADOS E DISCUSSÃO}

Os teores de vitamina $\mathrm{C}$ da mangaba recémcolhida do tipo "de caída" foram superiores, quando comparados aos das mangabas recém-colhidas do tipo "de vez" (Tabela 1). Comparando-se os teores de vitamina $\mathrm{C}$ da mangaba com outros frutos, tais como morango, goiaba, abacaxi, (ANDRADE et al., 2002), verifica-se que a mangaba se apresenta como um fruto com teor elevado de vitamina $\mathrm{C}$, ou seja, aproximadamente 252,7 a $274,7 \mathrm{mg}$ de Vit. C $100 \mathrm{~g}^{-1} \mathrm{MF}$. Diferença significativa nos teores de vitamina $\mathrm{C}$, de acordo com o estádio de maturação, também foi verificada por Melo et al. (2000) em pitanga e por Howad et al. (1994) em pimenta vermelha, a qual apresenta $30 \%$ mais vitamina $\mathrm{C}$, quando comparada à pimenta verde.

TABELA 1 - Características físco-químicas dos frutos recém-colhidos de mangaba "de caída" e "de vez" na região de Itaporanga D’Ajuda/SE.

\begin{tabular}{lcc}
\hline \multicolumn{1}{c}{ Parâmetro } & $\begin{array}{c}\text { Fruta } \\
\text { caída” "de }\end{array}$ & $\begin{array}{c}\text { Fruta } \\
\text { vez" "de }\end{array}$ \\
\hline Vitamina C & $274,7 \pm 8,0 \mathrm{a}^{1}$ & $252,7 \pm 7,5 \mathrm{~b}$ \\
Sólidos solúveis & $15,2 \pm 1,0 \mathrm{a}$ & $13,1 \pm 1,0 \mathrm{~b}$ \\
Acidez & $0,7 \pm 0,1 \mathrm{a}$ & $0,8 \pm 0,1 \mathrm{a}$ \\
$\mathrm{pH}$ & $3,5 \pm 0,1 \mathrm{a}$ & $3,5 \pm 0,2 \mathrm{a}$ \\
Atividade de Água & $0,988 \pm 0,005 \mathrm{a}$ & $0,986 \pm 0,006 \mathrm{a}$ \\
Condutividade Térmica & $0,05 \pm 0,04 \mathrm{a}$ & $0,04 \pm 0,01 \mathrm{a}$ \\
Resistividade Térmica & $26,9 \pm 10,9 \mathrm{a}$ & $30,2 \pm 7,8 \mathrm{a}$ \\
Difusividade Térmica & $0,17 \pm 0,04 \mathrm{a}$ & $0,18 \pm 0,04 \mathrm{a}$ \\
\hline
\end{tabular}

1-médias seguidas de mesma letra nas linhas não diferem estatisticamente a nível de $5 \%$ de probabilidade pelo teste T-Student.

Para frutos "de caída", observaram-se valores elevados de sólidos solúveis (Tabela 1). Os teores de sólidos solúveis foram superiores, quando comparados com outros frutos tropicais, como goiaba, cajá e pitanga, os quais apresentam, respectivamente, 7,$6 ; 8,5$; e 3,8
${ }^{\circ}$ Brix. (YAMASHITA e BENASSI, 2000; MELO et al., 2000; MOREIRA et al., 2002).

Não houve diferença significativa entre os valores de acidez total titulável (Tabela 1) determinados nas amostras em estudo. No entanto, os teores de acidez total titulável de mangaba foram de aproximadamente 0,72\% de ácido cítrico e pH de 3,5 (Tabela 1), apresentando valores intermediários quando relacionados com outros frutos, como goiaba, a qual apresenta $0,41 \%$ de ácido cítrico (YAMASHITA e BENASSI, 2000) e cajá, com1,56\% de ácido cítrico e PH de 2,9 (MOREIRA et al., 2002).

Em relação aos parâmetros físicos estudados, não houve diferença significativa entre as amostras de frutos "de vez" e "de caída" (Tabela 1). Para atividade de água, verificou-se uma semelhança entre os frutos "de vez" e "de caída". Observou-se tendência a valores menores na resistividade térmica do fruto "de caída", quando comparado aos frutos "de vez". Foi verificada, também, uma diferença entre os valores encontrados para a condutividade térmica e difusividade térmica.

Os teores de vitamina C (Figura 1 A) para mangaba "de vez" mantiveram-se entre 250 e $300 \mathrm{mg}$ de vit.C $100 \mathrm{~g}^{-1} \mathrm{MF}$, durante todo o período experimental, quando os frutos foram mantidos a $6^{\circ} \mathrm{C}$. Observou-se aumento nos teores de vitamina $\mathrm{C}$ até o terceiro dia de armazenamento, tanto para mangaba "de vez" quanto para "de caída", quando armazenadas a $25^{\circ} \mathrm{C}$. Vários autores observaram que frutos e hortaliças apresentam diminuição nos teores de vitamina $\mathrm{C}$ após a colheita e armazenamento (LEE e KADER, 2000). No entanto, alguns frutos cítricos e hortaliças podem apresentar uma retenção ou aumento nos teores de Vitamina C quando armazenados. Aspargo, por exemplo, apresenta aumento nos teores de vitamina $\mathrm{C}$ dois dias após a colheita, quando armazenados a $4^{\circ} \mathrm{C}$ (LEE e KADER, 2000). Vitamina $C$ também atua como um importante antioxidante (SMIRNOFF, 1995). Dessa forma, sugere-se que o aumento teores de vitamina $\mathrm{C}$ observado em frutos armazenados a $25^{\circ} \mathrm{C}$ (Figura $1 \mathrm{~A}$ ) pode estar relacionado à atuação da vitamina $\mathrm{C}$ como antioxidante, em resposta ao avanço das reações oxidativas que ocorrem durante o amadurecimento.

As mangabas "de caída" tornaram-se deterioradas após 3 e 7 dias de armazenamento a $25^{\circ} \mathrm{C}$ e $18^{\circ} \mathrm{C}$, respectivamente, apresentando sinais de deterioração e tornando impróprias para o consumo ou industrialização. Assim, as análises nessas temperaturas não tiveram continuidade após esse período. Após 5 dias de 
armazenamento dos frutos "de vez" mantidos a $6^{\circ} \mathrm{C}$ e 3 dias de armazenamento dos frutos "de caída" mantidos a $18^{\circ} \mathrm{C}$, verificou-se uma redução nos teores de Vitamina C. Esse comportamento também foi observado para outros frutos e hortaliças por Favell (1998) e Beaulieu et al. (1997). C. De acordo com Klein (1987), a diminuição dos teores de vitamina C está relacionada ao avanço do processo de amadurecimento e degradação dos frutos e ao aumento da temperatura. Assim, a vitamina C pode ser considerada um indicador da perda de qualidade dos frutos (KLEIN, 1987).

A $6^{\circ} \mathrm{C}$, a mangaba "de vez" apresentou índice de acidez de $0,7 \%$ ácido cítrico (Figura $1 \mathrm{~B}$ ) e pH de apro- ximadamente 3,5 (Figura 2 B). Mangabas "de caída" apresentaram valores de $\mathrm{pH}$ entre 2,97 e 4,56 nas três condições de temperatura estudadas (Figura 1, B).

Os teores de sólidos solúveis totais (Figura $2 \mathrm{~A}$ ) das amostras "de caída", mantidas à temperatura de $6^{\circ} \mathrm{C}$, variaram de 15,2 a $17,0{ }^{\circ}$ Brix e, para a fruta "de vez", de 13,1 a 15,67 'Brix. Observou-se também que quando armazenadas à temperatura de $18^{\circ} \mathrm{C}$, os frutos "de caída" apresentaram aumento nos teores de sólidos solúveis. Isso pode ter ocorrido devido à perda de água do fruto, que foi de $21 \pm 2 \%$ durante o período de armazenamento, concentrando o valor de ${ }^{\circ}$ Brix entre 15,2 e 18,37 .
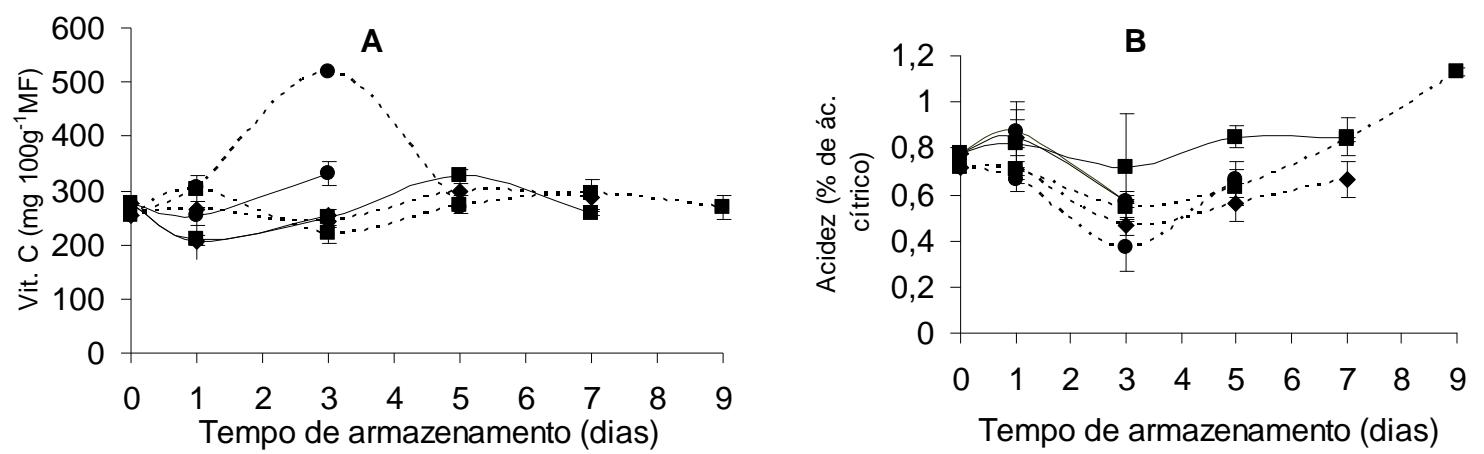

FIGURA 1 - Teores de vitamina C (A) e Acidez total titulável (B) da mangaba "de caída" (—) e "de vez" (--) armazenadas em diferentes temperaturas, $6^{\circ} \mathrm{C}(\boldsymbol{\square}), 18^{\circ} \mathrm{C}(\bullet)$ e $25^{\circ} \mathrm{C}(\bullet)$ durante o período de armazenamento.

A

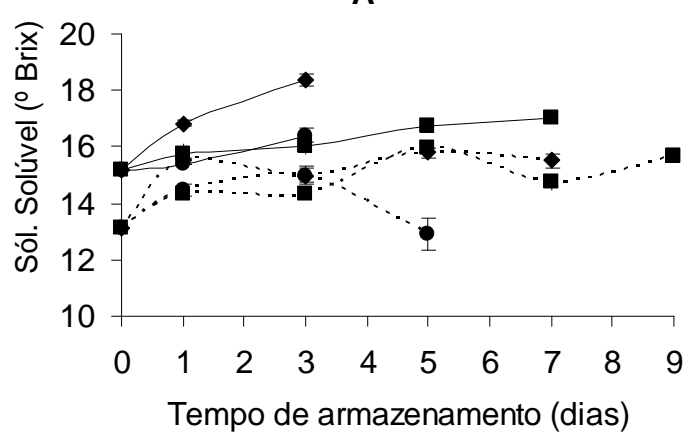

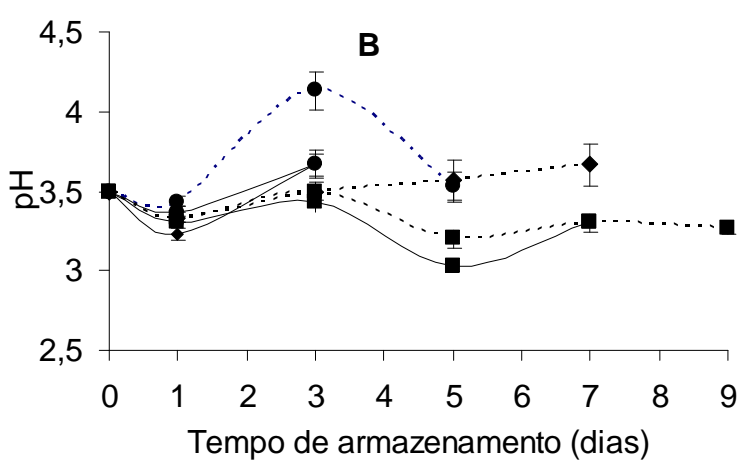

FIGURA 2 - Teores de sólidos solúveis (A) e pH (B) da mangaba "de caída" (-) e "de vez" (---) armazenadas em diferentes temperaturas, $6^{\circ} \mathrm{C}(\boldsymbol{\square}), 18^{\circ} \mathrm{C}(\bullet)$ e $25^{\circ} \mathrm{C}(\bullet)$, durante o período de armazenamento. 
$\mathrm{Na}$ temperatura de $25^{\circ} \mathrm{C}$ e a partir do terceiro dia de armazenamento, constatou-se perda na quantidade de sólidos solúveis de mangaba "de vez", em razão da degradação do produto com o passar do tempo.

Os valores de $\mathrm{pH}$ (Figura $2 \mathrm{~B}$ ) obtidos para a mangaba "de caída" variaram de 3,03 a 3,67 nas três diferentes temperaturas, 6,18 e $25^{\circ} \mathrm{C}$, durante o armazenamento. Para as temperaturas de 18 e $25^{\circ} \mathrm{C}$, foi observado um aumento no $\mathrm{pH}$ de 3,23 a 3,67 e, à temperatura de $6^{\circ} \mathrm{C}$, houve uma maior estabilidade nos resultados dessa variável. Resultados semelhantes foram observados para as mangabas "de vez".

Pelos resultados apresentados, verifica-se claramente que a temperatura de $6^{\circ} \mathrm{C}$ é eficiente em manter a qualidade dos frutos de mangaba "de vez", sendo indicada para o armazenamento dos frutos. No entanto, estudos devem ser realizados para verificar se após a retirada dos frutos da temperatura de $6^{\circ} \mathrm{C}$, eles completarão seu ciclo normal de amadurecimento, sem alterar suas características sensoriais.

Ao longo do período de armazenamento, observou-se perda de atividade de água tanto para mangaba "de vez" como para a "de caída", em todos os tratamentos (Figura $3 \mathrm{~A}$ ).

Sugere-se que a queda na Aw observada seja devida a um aumento nos teores de sólidos solúveis que ocorre durante o processo de amadurecimento dos frutos, como também à perda de peso dos frutos durante o armazenamento.

A difusividade térmica apresentou tendência de queda durante o armazenamento, independente do tipo de fruto; entretanto, essa variação não foi significativa (Figura 3 B).

Observou-se também que durante o tempo de armazenamento houve aumento na condutividade térmica (Figura $4 \mathrm{~A}$ ) e, consequientemente, uma diminuição da resistividade térmica (Figura 4 B) quando os frutos foram armazenados a $25^{\circ} \mathrm{C}$. Com esses resultados, verifica-se que o processo de amadurecimento dos frutos progride normalmente. Quando armazenados à temperatura de $6^{\circ} \mathrm{C}$, esses parâmetros não sofreram variação, como conseqüência da diminuição do metabolismo dos frutos submetidos à baixa temperatura, mantendo, assim, as suas características iniciais.

Sweat (1974) e Ribeiro et al. (2002) sugerem que as propriedades térmicas dos frutos podem ser utilizadas como parâmetros de acompanhamento das modificações metabólicas que ocorrem durante o processo de amadurecimento dos frutos. Para mangaba, verificou-se que os parâmetros condutividade térmica, resistividade e atividade de água podem ser utilizados como parâmetros de acompanhamento na pós-colheita dos frutos.
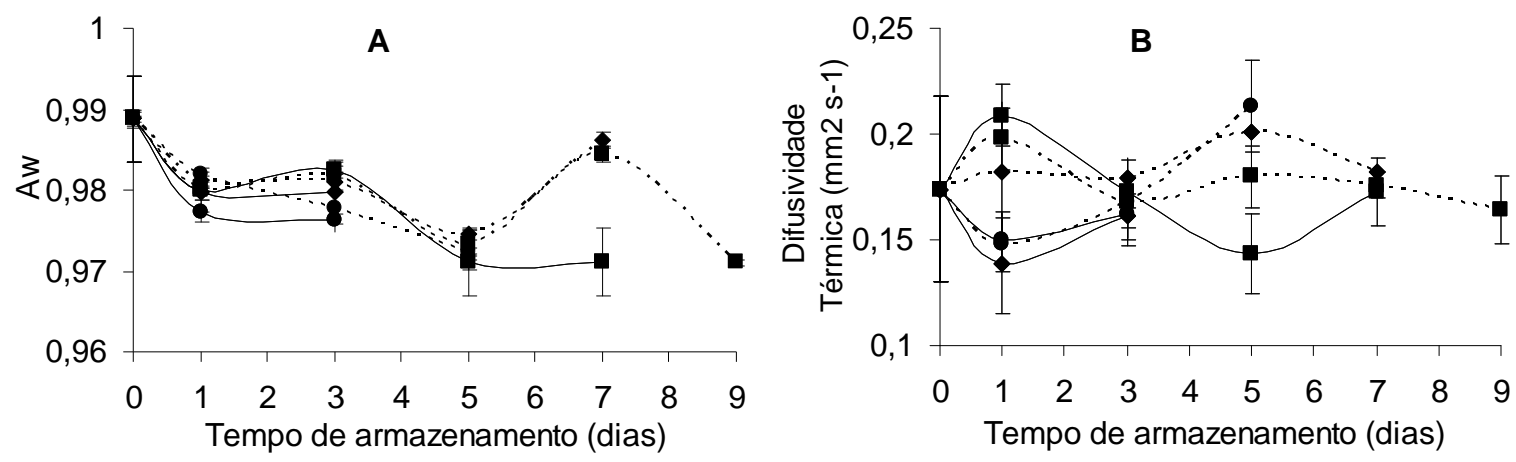

FIGURA 3 - Atividade de água Aw (A) e difusividade térmica (B) da mangaba "de caída" (-) e "de vez" (--) armazenadas em diferentes temperaturas, $6^{\circ} \mathrm{C}(\boldsymbol{\square}), 18^{\circ} \mathrm{C}(\bullet)$ e $25^{\circ} \mathrm{C}(\bullet)$, durante o período de armazenamento. 


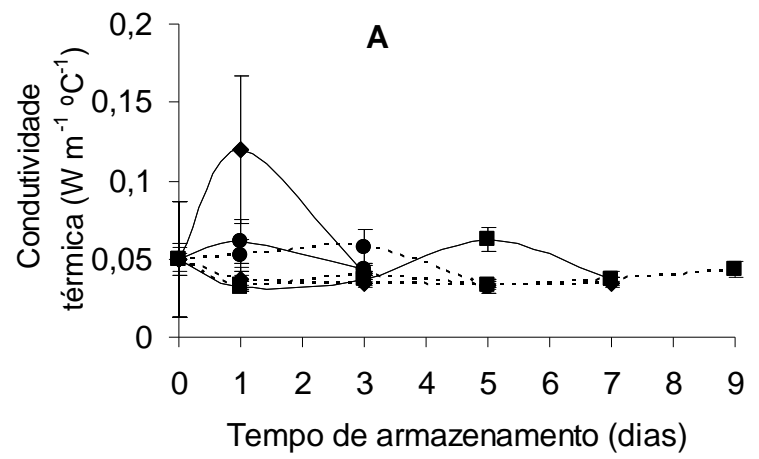

FIGURA 4 - Condutividade térmica (A) e resistividade (B) das em diferentes temperaturas, $6^{\circ} \mathrm{C}(\boldsymbol{\square}), 18^{\circ} \mathrm{C}(\bullet)$ e $25^{\circ} \mathrm{C}$

\section{CONCLUSÕES}

Mangabas "de caída" apresentaram, de modo geral, teores de vitamina $\mathrm{C}$, sólidos solúveis e atividade de água (Aw) superiores aos frutos "de vez".

Parâmetros físicos não diferiram significativamente entre si, tanto para frutos "de vez" como para frutos "de caída".

Os frutos de mangaba apresentaram teores de vitamina C em torno de 250 a $300 \mathrm{mg}$ Vit. C $100 \mathrm{~g}^{-1} \mathrm{MF}$ durante todo o período de armazenamento. Os teores de vitamina $\mathrm{C}$ podem ser utilizados como um indicativo de perda de qualidade dos frutos de mangaba.

$\mathrm{O}$ armazenamento sob refrigeração manteve a qualidade das mangabas "de caída" por um período de até três dias, quando submetidas às temperaturas de $18^{\circ} \mathrm{C}$ e $25^{\circ} \mathrm{C}$ e um período de até 7 dias, quando submetidas a uma temperatura de $6^{\circ} \mathrm{C}$.

A atividade de água (Aw) para mangabas "de vez" sofre queda durante o período de armazenamento refrigerado.

A condutividade térmica, a resistividade térmica e a atividade de água podem ser utilizadas como parâmetros de acompanhamento pós-colheita dos frutos de mangaba, quando armazenados sob refrigeração.

\section{AGRADECIMENTOS}

À FINEP, CNPq e EMDAGRO, pelo suporte financeiro para execução dos trabalhos.

\section{REFERÊNCIAS BIBLIOGRÁFICAS}

ANDRADE, R. S. G.; DINIZ, M. C. T.; NEVES, E. A.; NÓBREGA, J. A. Determinação e distribuição de ácido ascórbico em três frutos tropicais. Ecletica Quimica, Marília, v. 27, p. 393-401, 2002.

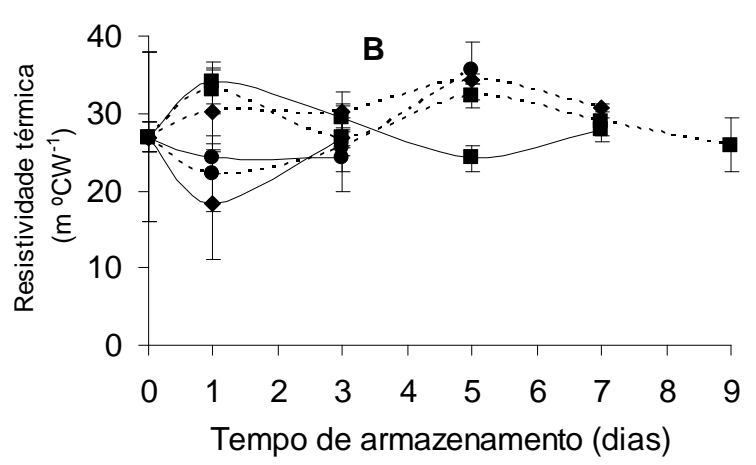

(B) da mangaba "de caída" (-) e "de vez" (---) armazena${ }^{\circ} \mathrm{C}(\bullet)$, durante o período de armazenamento.

BEAULIEU, J. C.; OLIVEIRA, F. A. R.; FERNANDES, T. D.; FONSEVCA, S. C.; BRECHT, J. K. Fresh-cut kale: quality assessment of portuguese storage-supplied product for development of a MPA system. CA'97 proceedings, [S.1.], v. 5, p. 145-151, 1997.

CARNELOSSI, M. A. G. Fisiologia pós-colheita de folhas de couve (Brassica oleracea cv. acephala) minimamente processadas. 2000. $81 \mathrm{f}$. Tese (Doutorado em Fisiologia Vegetal) - Universidade Federal de Viçosa, Viçosa, 2000.

CHITARRA, M. I. F.; CHITARRA, A. D. Pós-colheita de frutos e hortaliças: fisiologia e manuseio. Lavras: ESAL/FAEPE, 1990. 320 p.

FAGUNDES, G. R.; YAMANISHI, O. K. Características físicas e químicas de frutos de mamoeiro do grupo 'solo' comercializado em 4 estabelecimentos de Brasília - DF. Revista Brasileira de Fruticultura, Cruz das Almas, v. 23, n. 3, p. 541-545, 2001.

FAVELL, D. J. A comparison of the vitamin C content of fresh and frozen vegetables. Food Chemisty, London, v. 62, n. 1, p. 59-64, 1998.

HOWAD, L. R.; SMITH, R. T.; WAGNER, A. B.; VILLALON, B.; BURNS, E. E. Provitamin A and ascorbic acid content of fresh pepper cultivars (Capsicum annum) and processed jalapenos. Journal of Food Science, Chicago, v. 59, p. 362-365, 1994.

KLEIN, B. P. Nutritional consequences of minimal processing of fruits and vegetables. Journal Food Quality, Wastport, v. 10, p. 179-193, 1987. 
LEE S. K.; KADER, A. A. Preharvest and postharvest factors influencing vitamin $\mathrm{C}$ content of horticultural crops. Postharvest Biology and Tecnology, Amsterdam, v. 20 , p. $207-220,2000$.

MELO, E. A.; LIMA, V. L. A. G.; NASCIMENTO, P. Temperatura no armazenamento de pitanga. Scientia Agricola, Piracicaba, v. 57, n. 4, p. 629-634, 2000.

MOREIRA, M. A. B.; SOUZA, F. X.; RITZINGER, C. H. P.; RITZINGER, R.; FILGUEIRAS, H. A. C. Cajá (Spondias mombin L. Spondias lútea L.) In: VIEIRA NETO, R. D. (Ed.). Frutíferas potenciais para os tabuleiros costeiros e baixadas litorâneas. Aracaju: Embrapa, 2002. cap. 2, p. 21-44.

PAULL, R. E. Tropical fruit physiology and storage potential. In: CHAMP, B. R.; HIGHLEY, E.; JOHNSON, G. I. Phostharvest handling of tropical fruits. [S.1.: s.n.], 1994. p. 198-302.

RIBEIRO, V. S.; SOBRAL, M. C.; ALMEIDA, M. M.; SILVA, G. F. Propriedades físicas de produtos agrícolas. Revista Brasileira de Produtos Agroindustriais, [S.1.], v. 4, n. 1, p. 61-67, 2002.

SMIRNOFF, N. Environment and plant metabolism. Oxford: BIOS Scientific, 1995. 270 p.

SWEAT, V. E. Experimental values of thermal conductivity of selected fruits and vegetables. Journal of food Science, Chicago, v. 39, p. 10801083, 1974.

VIEIRA NETO, R. D. Frutíferas potenciais para os tabuleiros costeiros e baixadas litorâneas. Aracaju: Embrapa, 2002. 216 p.

YAMASHITA, F.; BENASSI, M. T. Influência da embalagem de atmosfera modificada e do tratamento com cálcio na cinética de degradação de ácido ascórbico e perda de peso em goiabas (Psidioum guajava L.). Ciência Tecnologia de Alimentos, Campinas, v. 20, n. 1, p. 27-31, 2000. 